\title{
VIWITHAN: A STANDARDIZED ASHWAGANDHA EXTRACT AMELIORATES OVALBUMIN- INDUCED AIRWAY-INFLAMMATION AND OXIDATIVE STRESS IN MOUSE MODEL
}

\author{
VASAVI HS*, SUDEEP HV, RAMANAIAH ILLURI, SHYAMPRASAD K
}

R and D Centre for Excellence, Vidya Herbs Private Limited, Bengaluru, Karnataka, India. Email: vasavitumri@gmail.com

Received: 23 October 2020, Revised and Accepted: 08 January 2021

\section{ABSTRACT}

Objective: Withania somnifera, commonly known as Ashwagandha, Indian ginseng, has been used in Ayurvedic and indigenous medicinal preparations for various disease conditions since long time. In the present study, we investigated the protective effects of Viwithan, a standardized proprietary extract from Ashwagandha roots, against airway-inflammation and oxidative stress modulation in an ovalbumin (OVA)-induced murine model of inflammation.

Methods: Allergic asthma was initiated in BALB/c mice by sensitizing with OVA on days 1 and 14, followed by intranasal challenge with OVA on days 27, 28, and 29. Mice were administered Viwithan (200 and $400 \mathrm{mg} / \mathrm{kg}$ ) by oral gavage before challenge. Then, mice were evaluated for the presence of airway inflammation, production of allergen-specific cytokine response, lung pathology, and oxidative stress modulation.

Results: The results showed that treatment with Viwithan attenuated OVA-induced lung inflammation in mice. Viwithan significantly attenuated inflammatory cell infiltration into the bronchoalveolar lavage fluid and markedly reduced the levels of pro-inflammatory cytokines, interleukin-10, and transforming growth factor- $\beta 1$ in lung tissues. Viwithan treatment considerably reduced the lung weight in OVA-sensitized mice. Viwithan markedly attenuated the OVA-induced generation of reactive oxygen species in lung tissues.

Conclusion: Together, these results suggested that Viwithan alleviates OVA-induced airway-inflammation and oxidative stress, highlighting the potential of standardized Ashwagandha extract as a useful therapeutic agent for pulmonary fibrosis management.

Keywords: Ashwagandha, Lung inflammation, Oxidative stress, Withaferin A, Withania somnifera.

(C) 2021 The Authors. Published by Innovare Academic Sciences Pvt Ltd. This is an open access article under the CC BY license (http://creativecommons.org/ licenses/by/4.0/) DOI: http://dx.doi.org/10.22159/ajpcr.2021v14i3.40119. Journal homepage: https://innovareacademics.in/journals/index.php/ajpcr

\section{INTRODUCTION}

Asthma is a complex and chronic inflammatory disorder of the lung tissue characterized by the infiltration of inflammatory cells, airway inflammation, bronchial hyper-responsiveness, and mucus hypersecretion [1,2]. The incidence and prevalence of asthma is rapidly increasing around the world and it has become a significant cause of morbidity and mortality in developed countries [3]. A few patients suffering from airway inflammation develop honeycomb lung and suffer mortality due to the irreversible loss of pulmonary function [4]. Airway inflammation results in the upregulation of a large variety of reactive oxygen species (ROS) in the lungs, thereby causing oxidative damage to the airways. Oxidative stress can initiate and propagate airway inflammation, highlighting the importance of pro-oxidants in inflammatory airway disorders [5]. Eosinophils are well known for their contribution to inflammation in airway disorders through the release of ROS and specific granules [6]. Recent research has been focusing on exploration of various drugs for the treatment of airway inflammation; however, they are not particularly effective and more often accompanied by adverse side effects during long-term treatment [7].

Medicinal plants provide an extensive resource in the search for effective medical treatments with a low risk of adverse effects. Recently, herbal preparations have drawn greater attention for treating respiratory diseases, including airway inflammation, asthma, cystic fibrosis, and chronic bronchitis $[8,9]$. Withania somnifera (Solanaceae) commonly known as Ashwagandha, Indian ginseng, is an important medicinal plant used long since in indigenous system of medicine including Ayurveda. Ashwagandha provides benefits against many human illnesses such as epilepsy, depression, arthritis, diabetes, and palliative effects such as analgesic, rejuvenating, regenerating, and growth-promoting effects $[10,11]$. Phytosteroids such as withaferin A and withanoloids are the main biologically active compounds and most of the therapeutic properties of Ashwagandha are attributed to them. These compounds are reported to have anti-inflammatory, immunomodulatory and antitumor properties [12].

Previous studies conducted in animal models showed that withaferin A could attenuate brain injury by suppressing inflammation [13]. In addition, withaferin A has been reported as an effective treatment to reduce the progression of fibrosis [14]. Therefore, it is hypothesized that withaferin A may also be a potential candidate for the treatment of airway inflammation. Hence, the present investigation is aimed to assess the therapeutic efficacy of Viwithan, a proprietary extract of Ashwagandha roots containing 5\% withaferin A in ovalbumin (OVA)induced airway-inflammation model.

\section{METHODS}

\section{Preparation of plant extract}

Viwithan $^{\mathrm{TM}}$, a proprietary extract of Ashwagandha roots containing 5\% withaferin A was obtained from the Department of Quality control and Assurance, Vidya Herbs Private Limited, Bengaluru.

Animals

Thirty healthy male BALB/c mice weighed 25-35 g were purchased from commercial animal breeder, Biogen Private Limited, Bengaluru, India. Mice were kept for acclimatization, 7 days before dosing. Animals were housed in groups of five per standard cage, on $12 \mathrm{~h}$ light/dark cycle; and air temperature was maintained at $22 \pm 2^{\circ} \mathrm{C}$. Experiments were carried out in accordance with guidelines and approved by Institutional Animal Ethical Committee of Vidya Herbs Private Limited, Bengaluru. 
The mice were randomly divided into five groups $(n=6)$ : (i) Group I: Normal control group; (ii) Group II: Treated with OVA; (iii) Group III: Treated with OVA and dexamethasone (2 mg/kg); (iv) Group IV: Treated with OVA and Viwithan $200 \mathrm{mg} / \mathrm{kg}$; and (v) Group IV: Treated with OVA and Viwithan $400 \mathrm{mg} / \mathrm{kg}$.

\section{Experimental protocol}

The airway-inflammation was induced by OVA (Sigma-Aldrich) using the method described previously [15]. Briefly, all the mice except normal control group were sensitized by intraperitoneal (i.p.) injection of $50 \mu \mathrm{g}$ of OVA with $1 \mathrm{mg}$ of aluminum hydroxide (Sigma-Aldrich) on day $1^{\text {st }}$ and day $14^{\text {th }}$. The OVA-challenged mice were exposed to intranasal challenge with $2 \%$ OVA solution from days $27^{\text {th }}$ to $29^{\text {th }}$ after the second sensitization. Group IV and Group V of asthma mice were orally administrated once daily with $200-400 \mathrm{mg} / \mathrm{kg}$ of Viwithan, respectively, and Group III was treated with dexamethasone $2 \mathrm{mg} / \mathrm{kg}$ daily, from days 15 to 26 . Normal control group was only sensitized and challenged with phosphate buffered saline (PBS). Mice were sacrificed to determine the pathophysiological features of asthma $24 \mathrm{~h}$ after the last challenge.

\section{Cytokine analysis}

The levels of interleukin-10 (IL-10) and transforming growth factor- $\beta 1$ (TGF- $\beta 1$ ) in the lung tissue homogenates were estimated using commercially available enzyme-linked immunosorbent assay (ELISA) kit (USCN Life science Inc., Wuhan, China) according to the manufacturer's protocol.

\section{Histological analysis}

The lung tissue samples were embedded in paraffin and stained with hematoxylin and eosin to examine the histological changes. Photomicrographs were taken using a light microscope.

\section{Inflammatory cell infiltration in the bronchoalveolar lavage fluid (BALF)}

Following treatment with OVA and Viwithan, the mice in each group were sacrificed and their BALF was collected by washing lungs 3 times with chilled PBS using a tracheal cannula. The BALF samples were then centrifuged at $\times 1000 \mathrm{~g}$ for $10 \mathrm{~min}$ at $4^{\circ} \mathrm{C}$ and the cell pellet was resuspended in PBS. The total BALF cell number was determined using a hemocytometer. The cells were then cytospin onto microscope slides and stained with Wright-Giemsa (50\% in PBS) using a cytospin for total $6 \mathrm{~min}$ at room temperature for cell classification. The percentages of BALF neutrophils, macrophages, and lymphocytes were obtained by counting the leukocytes under light microscopy at $\times 400$.

\section{Estimation of reduced glutathione (GSH) content}

GSH level was determined by the method of Ellman (1959) with slight modification [16]. Briefly, $100 \mu \mathrm{l}$ of lung homogenate was mixed with $100 \mu \mathrm{l}$ of $10 \mathrm{mM}$ Ellman's reagent. The volume was made up to $1 \mathrm{ml}$ using $0.2 \mathrm{M}$ Tris buffer with $1 \mathrm{mM}$ ethylenediaminetetraacetic acid (EDTA), pH 8.2 and the yellow color developed was read at $412 \mathrm{~nm}$. The concentration of GSH in the sample was determined by calculating from the linear equation or the regression curve generated from GSH standard.

\section{Superoxide dismutase (SOD) activity}

For total SOD assay, $3.0 \mathrm{ml}$ reaction mixture contained $50 \mathrm{mM}$ sodium carbonate ( $\mathrm{pH}$ 10.2), $96 \mu \mathrm{M}$ nitroblue tetrazolium (NBT), $0.1 \mathrm{mM}$ EDTA, $20 \mathrm{mM}$ hydroxylamine hydrochloride, $0.6 \%$ (v/v) Triton X-100, and $50 \mu \mathrm{l}$ lung homogenate. The absorbance was recorded at $560 \mathrm{~nm}$ for 5 min. One unit of SOD activity was defined as the amount of enzyme required that caused $50 \%$ of $\mathrm{NBT}$ reduction at $25^{\circ} \mathrm{C}$ [17].

\section{Lipid peroxidation assay}

Lipid peroxidation in the lung homogenate was measured by estimating the formation of thiobarbituric acid reactive substances (TBARS). Malondialdehyde (MDA) is an end product of lipid peroxidation, which reacts with thiobarbituric acid to form pink chromogen TBARS [18]. The reaction mixture contained $0.1 \mathrm{ml}$ of lung homogenate, $0.9 \mathrm{ml}$ of $10 \%$ trichloroacetic acid, and $1.0 \mathrm{ml}$ of $0.8 \%$ thiobarbituric acid in $20 \%$ acetic acid. Solution was incubated at $95^{\circ} \mathrm{C}$ in water bath for $60 \mathrm{~min}$. After incubation, solution was cooled, and absorbance was measured at $532 \mathrm{~nm}$. Results were expressed as $\mu \mathrm{mol} \mathrm{MDA} / \mathrm{mg}$ protein.

\section{RESULTS}

Viwithan administration ameliorates OVA-induced lung injury by suppressing pulmonary fibrosis in mice

There was no significant difference in body weight observed in all the treatment groups following the OVA-challenge compared with the control group. The lung weight of the OVA-challenged mice slightly increased compared to that in the control group. In contrast, treatment with Viwithan $(200 \mathrm{mg} / \mathrm{kg})$ slightly decreased the lung weight. Our results suggest that Viwithan inhibited the OVA-induced lung weight gain in airway tissues (Table 1).

Viwithan reduced the number of inflammatory cells in BALF

BALF was collected $24 \mathrm{~h}$ after the last OVA-challenge; the effect of Viwithan on OVA-induced total cells and leukocytes (i.e., macrophages, lymphocytes, basophils, and eosinophils) was evaluated in BALF. OVA administration markedly increased the total cell count and the number of differential leukocytes in BALF, which were significantly suppressed by Viwithan compared with that of the control, suggesting that the inflammation leading to fibrosis was alleviated by Viwithan (Fig. 1).

Effects of Viwithan on the level of pro-inflammatory cytokines in the lung tissues of OVA-challenged mice

The levels of pro-inflammatory cytokines in response to OVAsensitization were measured in lung tissue homogenates by ELISA. The OVA-challenged mice showed a marked elevation of IL-10 and TGF- $\beta 1$ secretion compared with that for the PBS-treated controls. Treatment with Viwithan and dexamethasone suppressed the production of IL-10 and TGF- $\beta 1$ compared with those in the OVA group though it was not significant (Fig. 2). However, Viwithan at the concentration of $400 \mathrm{mg} / \mathrm{kg}$ showed a slight increase in the TGF- $\beta 1$ levels than the OVA group.

Viwithan ameliorated pathological changes of lung tissues in airway-inflammation model

In the present study, we investigated the effects of Viwithan on the morphological changes of lung tissues in asthma mice. A clear bronchitis was observed in OVA-induced mice which were characterized by epithelial and goblet cell hyperplasia, extensive fibrosis, mucous secretion, and alveolar hyperplasia with inflammation (Fig. 3B). However, these pathological changes were hardly observed in the

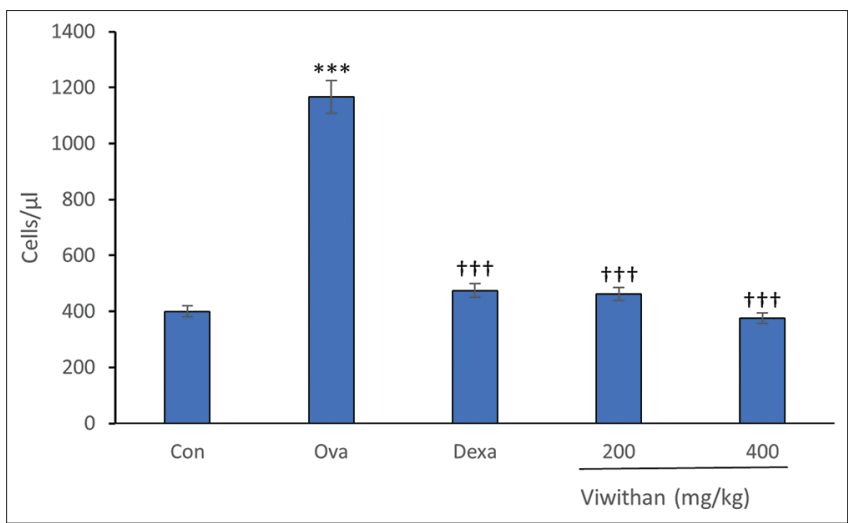

Fig. 1: Effect of Viwithan on inflammatory cells response in airway-inflammation model. Values are expressed as mean $\pm \mathrm{SD}$, $\mathrm{n}=6 .{ }^{*}$ Significant difference when compared with control group $(p<0.05)$. $\uparrow$ Significant difference when compared with ovalbumin group $(p<0.05)$ 
Table 1: Effect of Viwithan on body weight and lung weight in airway-inflammation model

\begin{tabular}{|c|c|c|c|c|c|c|c|}
\hline \multirow[t]{2}{*}{ Groups } & \multicolumn{5}{|c|}{ Body weight (g) } & \multirow[t]{2}{*}{ Lung weight (mg) } & \multirow[t]{2}{*}{ Relative Lung weight (mg) } \\
\hline & $\mathbf{0}$ & 7 & 14 & 21 & 28 & & \\
\hline Control & 30.98 & 31.38 & 32.37 & 32.15 & 32.55 & 275.38 & 847.27 \\
\hline OVA + Dexamethasone & 33.42 & 33.67 & 35.45 & 33.78 & 33.64 & 287.38 & 861.33 \\
\hline OVA + Viwithan $(200 \mathrm{mg} / \mathrm{kg})$ & 33.66 & 35.18 & 36.02 & 35.85 & 36.31 & 326.43 & 891.50 \\
\hline OVA + Viwithan $(400 \mathrm{mg} / \mathrm{kg})$ & 33.19 & 33.87 & 35.06 & 34.45 & 34.19 & 343.13 & 1057.37 \\
\hline
\end{tabular}

OVA: ovalbumin

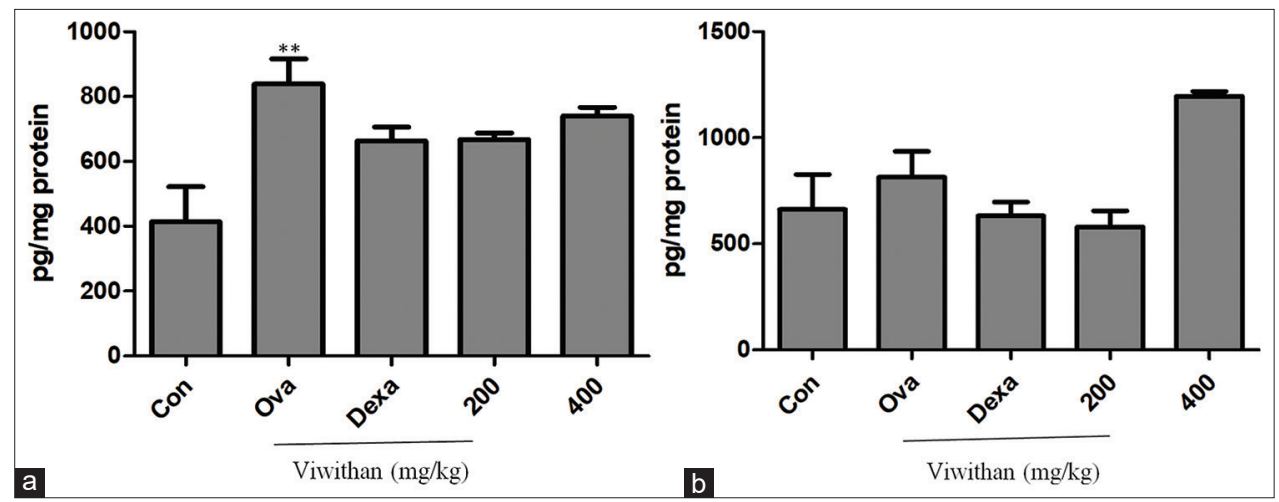

Fig. 2: Effect of Viwithan on pro-inflammatory cytokine levels in the lung tissues. (a) Interleukin-10 and (b) transforming growth factor- $\beta 1$. Values are expressed as mean $\pm S D, n=6$. *Significant difference when compared with control group ( $p<0.05$ )

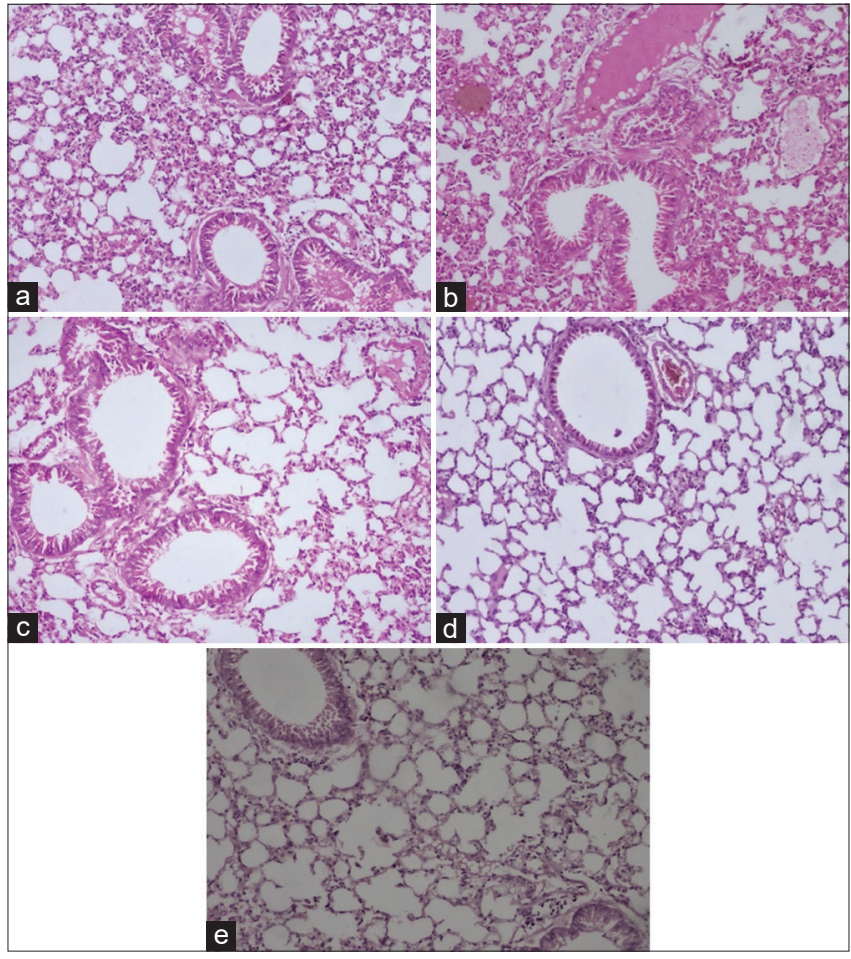

Fig. 3: Effect of Viwithan on ovalbumin (OVA)-induced histopathological alterations in the lung tissues. (a) Control; (b) OVA; (c) OVA+ Dexamethasone; (d) OVA + Viwithan (200 mg/ $\mathrm{kg})$; and (e) OVA + Viwithan (400 mg/kg)

control group (Fig. 3A). Treatment with dexamethasone (Fig. 3C) and Viwithan (Fig. 3D and E) reversed OVA-induced allergic inflammation response around the bronchial trees. Treated mice showed reduced leukocyte inflammatory infiltration evidenced by near normal appearance of alveoli and mild bronchiole epithelial hyperplasia.
Effect of Viwithan on oxidative stress induced by OVA in lung tissues

In this study, we examined the inhibitory effect of Viwithan on ROS production in lung tissues from OVA-challenged mice. GSH level was significantly increased in the treated groups compared to OVA-induced group (Fig. 4A). The concentration of MDA in the OVA-induced group was significantly higher $(\mathrm{p}<0.05)$ than the control group and all treatment groups significantly $(\mathrm{p}<0.05)$ reduced the MDA levels in the lung tissues (Fig. 4B). SOD activity was also significantly increased in the Viwithan treated group compared to OVA-induced group (Fig. 4C).

\section{DISCUSSION}

Ashwagandha (W. somnifera) root powder is being used in Ayurvedic system of medicine and remedies that have been in practice in India for 1000 years. Toxicological studies have found that, Ashwagandha, in its reasonable dose, is a non-toxic, safe, and edible herb [19]. Administration of hydroalcoholic extract of Ashwagandha containing $4.5 \%$ withaferin A showed no evidence of toxic effects or mortality up to the concentration of $2000 \mathrm{mg} / \mathrm{kg} /$ day for 28 days in Wistar rats [20]. Standardized extract of Ashwagandha and withaferin A has been found to be promising for their anticancer activity and immunomodulatory effects in animal models [21,22]. In the present study, using a mouse model of OVA-induced airway inflammation, we investigated the protective effects of Viwithan, a standardized extract of Ashwagandha containing 5\% withaferin A against allergic airway-inflammation. Results demonstrated that the treatment with Viwithan $(200 \mathrm{mg} / \mathrm{kg})$ protected against OVA-induced airway-inflammation by regulating inflammatory cytokines, reducing lung pathological alterations and oxidative stress.

Inflammation of the airway epithelium has typically been considered as the first defensive barrier, which minimizes allergen access to the body [23]. OVA has been demonstrated to induce airway inflammation in animal models for experiments [24]. The airway-inflammation induced in the present study model was characterized by excessive airway eosinophils and other inflammatory cell infiltration, goblet cell hyperplasia with excessive airway mucus, and elevated cytokines. 


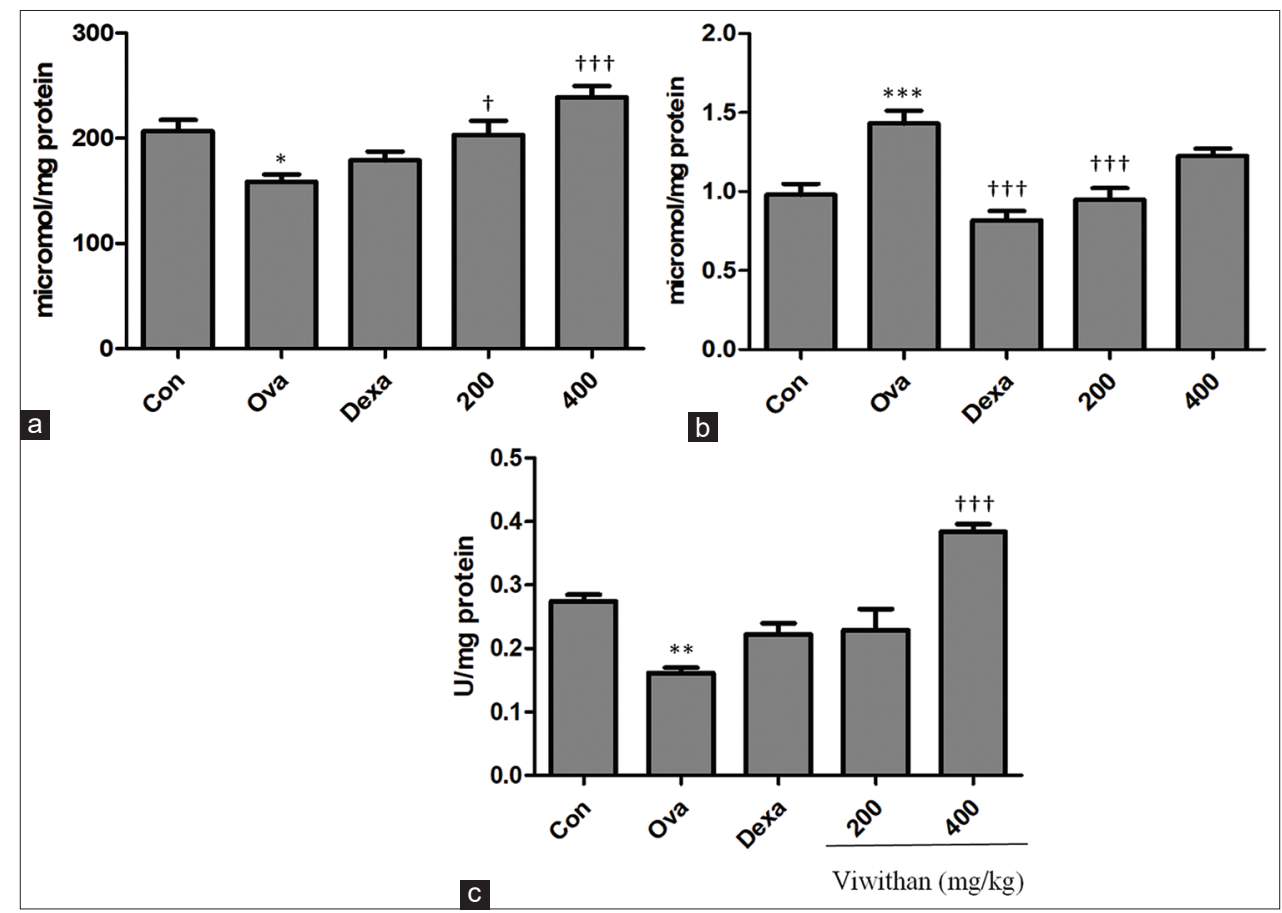

Fig. 4: Effect of Viwithan on oxidative stress induced in the lung tissues in airway inflammation model. (a) Superoxide dismutase; (b) malondialdehyde; and (c) glutathione. Values are expressed as mean $\pm \mathrm{SD}, \mathrm{n}=6 .{ }^{*}$ Significant difference when compared with control group $(p<0.05)$. †Significant difference when compared with ovalbumin group $(p<0.05)$

Furthermore, inflammatory immune cells, including eosinophils, neutrophils, monocytes, and epithelial cells, generate high levels of ROS to damage lung tissues [25,26]. Hence, asthma progression may be ameliorated by regulating the activity of cytokines and reducing the infiltration of inflammatory immune cells. Viwithan treatment evidently lowered cytokines and inflammatory cells especially eosinophils in BALF and lung homogenates.

Previous studies have indicated that pro-inflammatory cytokines, including IL-10 and TGF- $\beta 1$, are important in the pulmonary inflammatory response $[27,28]$. TGF- $\beta 1$ is associated with pulmonary fibrosis, during which it initiates fibroblast differentiation into myofibroblasts, and subsequently causes excessive collagen accumulation and extracellular matrix deposition [29]. TGF- $\beta 1$ also known to promote epithelial mesenchymal transition (EMT) during fibrotic diseases. EMT induced by TGF- $\beta 1$ is a key issue in the pathogenesis of tissue or organ fibrosis; it has been reported that the inhibition of TGF- $\beta 1$ may reverse EMT in hepatic fibrogenesis [30]. Therefore, suppression of TGF- $\beta 1$ is potentially beneficial for the treatment of airway inflammation and pulmonary fibrosis. In the present study, TGF- $\beta 1$ level has been increased in OVA-induced animals compared to normal control and treatment with Viwithan significantly reduced the TGF- $\beta 1$ level at the concentration of 200 $\mathrm{mg} / \mathrm{kg}$. Similarly, the increased levels of IL-10 in OVA-induced mice were significantly decreased after treatment with Viwithan. These results were in agreement with the previous reports where some plant extracts alleviated airway inflammation by regulating the production of inflammatory cytokines [31,32]. Our results also showed that Viwithan significantly attenuated the infiltration of eosinophils and other inflammatory cells.

Oxidative stress is closely associated with allergic and immunological disorders, such as asthma, atopic dermatitis, and allergic rhinitis [33]. Studies have shown that increased ROS production in allergic respiratory diseases contributes, in part, to tissue damage at sites of inflammation [34]. MDA is the product of lipid peroxidation and a classic indicator of oxidative stress and SOD is one of the major enzymes responsible for the inactivation of superoxide and hydrogen peroxide [35]. GSH, reduced GSH, is a key antioxidant responsible for primary defense system for protection from oxidative damage [36]. In the present study, Viwithan treatment significantly reduced oxidative stress induced by OVA-sensitization as indicated by decrease in MDA levels and increase in SOD activity and GSH levels. The results suggested that Viwithan has an antioxidant activity and is responsible for the decrease in the production of ROS, which finally may inhibit the tissue damage caused due to oxidative stress. Previous reports also support the potential antioxidant activity of Ashwagandha in various disease conditions [37].

\section{CONCLUSION}

Collectively, we demonstrated that treatment with Viwithan has a significant anti-inflammatory effect in allergic asthma and may prove to be an effective therapeutic reagent for airway-inflammation by suppressing pro-inflammatory cytokines, oxidative stress, and inflammatory cell infiltration. The present study warrants further evaluation at the molecular level for the treatment of airway inflammation.

\section{AUTHORS' CONTRIBUTIONS}

All authors have contributed equally.

\section{CONFLICTS OF INTEREST}

No conflicts of interest disclosed.

\section{AUTHORS' FUNDING}

This study was funded by Vidya Herbs Pvt. Ltd.

\section{REFERENCES}

1. Larsen GL, Patrick GH. The concept of airway inflammation. Am J Respir Crit Care Med 2000;162:S2-6.

2. Yuan M, Ge A, Zhu W, Liu YN, Ji NF, Zha WJ, et al. Morin attenuates ovalbumin-induced airway inflammation by modulating oxidative stress-responsive MAPK signaling. Oxid Med Cell Longev 2016;2016:5843672 
3. Anandan C, Nurmatov U, Schayck OC, Sheikh A. Is the prevalence of asthma declining? Systematic review of epidemiological studies. Allergy 2010;65:152-67.

4. Pouliot P, Camateros P, Radzioch D, Lambrecht BN, Olivier M. Protein tyrosine phosphatases regulate asthma development in a murine asthma model. J Immunol 2009;182:1334-40.

5. Qu J, Li Y, Zhong W, Gao P, Hu C. Recent developments in the role of reactive oxygen species in allergic asthma. J Thorac Dis 2017;9:E32-43.

6. Tashkin DP, Wechsler ME. Role of eosinophils in airway inflammation of chronic obstructive pulmonary disease. Int J Chron Obstruct Pulmon Dis 2018;13:335-49.

7. Yang X, Zhu J, Tung CY, Gardiner G, Wang Q, Chang HC, et al. Lunasin alleviates allergic airway inflammation while increases antigen-specific tregs. PLoS One 2015;10:e0115330.

8. Li S, Miao Z, Tian Y, Wang H, Wang S, He T, et al. Limethason reduces airway inflammation in a murine model of ovalbumin-induced chronic asthma without causing side effects. Exp Ther Med 2018;15:2269-76.

9. Kim HP, Lim H, Kwon YS. Therapeutic potential of medicinal plants and their constituents on lung inflammatory disorders. Biomol Ther 2017;25:91-104.

10. Singh N, Bhalla M, Jager P, Gilca M. An overview on ashwagandha: A rasayana (rejuvenator) of Ayurveda. Afr J Tradit Complement Altern Med 2011:8:208-13.

11. Jana SN, Charan SM. Health benefits and medicinal potency of Withania somnifera: A review. Int J Pharm Sci Rev Res 2018:48:22-9.

12. Verma SK, Kumar A. Therapeutic uses of Withania somnifera (Ashwagandha) with a note on withanolides and its pharmacological actions. Asian J Pharm Clin Res 2011;4:1-4.

13. Patel P, Julien JP, Kriz J. Early-stage treatment with Withaferin A reduces levels of misfolded superoxide dismutase 1 and extends lifespan in a mouse model of amyotrophic lateral sclerosis. Neurotherapeutics 2015;12:217-33.

14. Oh JH, Lee TJ, Park JW, Kwon TK, Withaferin A inhibits iNOS expression and nitric oxide production by Akt inactivation and downregulating LPS-induced activity of NF- $\kappa$ B in RAW 264.7 cells. Eur J Pharmacol 2008;599:11-7.

15. Piao CH, Bui TT, Song CH, Shin HS, Shon DH, Chai OH. Trigonella foenum-graecum alleviates airway inflammation of allergic asthma in ovalbumin-induced mouse model. Biochem Biophys Res Commun 2017;482:1284-8.

16. Ellman CL. Tissue sulfhydryl groups. Arch Biochem Biophys $1959 ; 82: 70-7$

17. Uchio R, Higashi Y, Kohama Y, Kawasaki K, Hirao T, Muroyama K, et al. A hot water extract of turmeric (Curcuma longa) suppresses acute ethanol-induced liver injury in mice by inhibiting hepatic oxidative stress and inflammatory cytokine production. J Nutr Sci 2017:6:e3.

18. Ohkawa H, Ohishi N, Yagi K. Assay for lipid peroxides in animal tissues by thiobarbituric acid reaction. Anal Biochem 1979;95:351-8.

19. Navi K, Saxena N, Shah N, Singh R, Manjunath K, Uthayakumar M, et al. Differential activities of the two closely related withanolides, Withaferin A and Withanone: Bioinformatics and experimental evidences. PLoS One 2012;7:e44419.

20. Shruti BP, Nirav JR, Hingorani LL. Safety assessment of Withania somnifera extract standardized for Withaferin A: Acute and sub-acute toxicity study. J Ayurveda Integr Med 2016;7:30-7.
21. Schliebs R, Liebmann A, Bhattacharya SK, Kumar A, Ghosal S, Bigl V. Systemic administration of defined extracts from Withania somnifera (Indian Ginseng) and Shilajit differentially affects cholinergic but not glutamatergic and GABAergic markers in rat brain. Neurochem Int 1997;30:181-90.

22. Grover A, Priyandoko D, Gao R, Shandilya A, Widodo N, Bisaria VS, et al. Withanone binds to mortalin and abrogates mortalin-p53 complex: Computational and experimental evidence. Int J Biochem Cell Biol 2012;44:496-504.

23. Koppelman GH. Gene by environment interaction in asthma. Curr Allergy Asthma Rep 2006;6:103-11.

24. Chen H, Xu X, Teng J, Cheng S, Bunjhoo H, Cao Y, et al. CXCR4 inhibitor attenuates ovalbumin-induced airway inflammation and hyperresponsiveness by inhibiting Th17 and Tc17 cell immune response. Exp Ther Med 2016;11:1865-70.

25. Saglani S, Lloyd CM. Eosinophils in the pathogenesis of paediatric severe asthma. Curr Opin Allergy Clin Immunol 2014;14:143-8.

26. Nakagome K, Matsushita S, Nagata M. Neutrophilic inflammation in severe asthma. Int Arch Allergy Immunol 2012;158:96-102.

27. Ogawa Y, Duru EA, Ameredes BT. Role of IL-10 in the resolution of airway inflammation. Curr Mol Med 2008;8:437-45.

28. Branchett WJ, Lloyd CM. Regulatory cytokine function in the respiratory tract. Mucosal Immunol 2019;12:589-600.

29. Lu Q. Transforming growth factor- $\beta 1$ protects against pulmonary artery endothelial cell apoptosis via ALK5. Am J Physiol Lung Cell Mol Physiol 2008;295:123-33.

30. Thakur S, Viswanadhapalli S, Kopp JB, Shi Q, Barnes JL, Block K, et al. Activation of AMP activated protein kinase prevents TGF- $\beta 1$-induced epithelial-mesenchymal transition and myofibroblast activation. Am J Pathol 2015;185:2168-80.

31. Yu B, Cai W, Zhang HH, Zhongc YS, Fang J, Zhang WY, et al. Selaginella uncinata flavonoids ameliorated ovalbumin-induced airway inflammation in a rat model of asthma. J Ethnopharmacol 2017;195:71-80

32. Lee JW, Min JH, Kim MG, Kim SM, Kwon OK, Oh TK, et al. Pistacia weinmannifolia root exerts a protective role in ovalbumin-induced lung inflammation in a mouse allergic asthma model. Int $\mathrm{J}$ Mol Med 2019;44:2171-80.

33. Sahiner UM, Birben E, Erzurum S, Sackesen C, Kalayci O. Oxidative stress in asthma. World Allergy Organ J 2011;4:151-8

34. Huang WC, Fang LW, Liou CJ. Phloretin attenuates allergic airway inflammation and oxidative stress in asthmatic mice. Front Immunol 2017;8:582838.

35. Liu YJ, Cao LP, Du JL, Jia R, Wang JH, Xu P, et al. Protective effects of Lycium barbarum polysaccharides against carbon tetrachlorideinduced hepatotoxicity in precision-cut liver slices in vitro and in vivo in common carp (Cyprinus carpio L.). Comp Biochem Physiol C Toxicol Pharmacol 2015;169:65-72.

36. Jiang WJ, Luo F, Lu QF, Liu JY, Li PJ, Wang XF, et al. The protective effect of Trillin LPS-induced acute lung injury by the regulations of inflammation and oxidative state. Chem Biol Interact 2016;243:127-34.

37. Mishra LC, Singh BB, Dagenais S. Scientific basis for the therapeutic use of Withania somnifera (Ashwagandha): A review. Altern Med Rev 2000;5:334-46. 